\title{
Complex tensor factorisation with PARAFAC2 for the estimation of brain connectivity from the EEG
}

\author{
Loukianos Spyrou*, Mario Parra, and Javier Escudero* \\ ${ }^{*}$ School of Engineering, Institute for Digital Communications, The University of Edinburgh, Alexander Graham \\ Bell Building, EH9 3FG, UK.
}

\begin{abstract}
Objective: The coupling between neuronal populations and its magnitude have been shown to be informative for various clinical applications. One method to estimate functional brain connectivity is with electroencephalography (EEG) from which the cross-spectrum between different sensor locations is derived. We wish to test the efficacy of tensor factorisation in the estimation of brain connectivity.

Methods: An EEG model in the complex domain is derived that shows the suitability of the PARAFAC2 model. Complex tensor factorisation based on PARAFAC2 is used to decompose the EEG into scalp components described by the spatial, spectral, and complex trial profiles. A connectivity metric is also derived on the complex trial profiles of the extracted components.

Results: Results on a benchmark EEG dataset confirmed that PARAFAC2 can estimate connectivity better than traditional tensor analysis such as PARAFAC within a range of signal-tonoise ratios. MVAR-ICA outperformed PARAFAC2 for very low signal-to-noise ratios while being inferior in most of the range, and in contrast to our method MVAR-ICA does not allow the estimation of trial to trial information. The analysis of EEG from patients with mild cognitive impairment or Alzheimer's disease showed that PARAFAC2 identifies loss of brain connectivity agreeing with prior pathological knowledge.

Conclusion: The complex PARAFAC2 algorithm is suitable for EEG connectivity estimation since it allows to extract meaningful coupled sources and provides better estimates than complex PARAFAC and MVAR-ICA.

Significance: A new paradigm that employs complex tensor factorisation has demonstrated to be successful in identifying brain connectivity and the location of couples sources for both a benchmark and a real-world EEG dataset. This can enable future applications and has the potential to solve some the issues that deteriorate the performance of traditional connectivity metrics.
\end{abstract}

Index Terms-complex tensor factorisation, PARAFAC2, connectivity, EEG

\section{INTRODUCTION}

Tensor factorisation has found many applications in several areas such as antenna array processing, blind source separation, biomedical signal processing, feature extraction, and classification [1], [2], [3], [4]. A tensor is a multiway representation of data or a multidimensional array. Each dimension in the tensor is called a mode or a way. Using tensor factorisation, the true underlying structure of that data can be revealed. Tensor factorisation methods have been shown to be powerful for describing signals which in general change in time, frequency, and space. Tensor analysis can provide a good way to discover the main features of the data and extract the

This work was supported by EPSRC, UK, Grant No. EP/N014421/1. hidden underlying information especially in the case of having big data size.

Several tensor based methods have been suggested for decomposition and multi-way representation of data. The PARAFAC decomposition [1], [2], [5] is one of the common tensor factorisation methods which is a generalisation of singular value decomposition (SVD) to higher order tensors. Using the PARAFAC model, the data are decomposed into a sum of rank-1 tensors of lower dimensions than the original data. Therefore, as suggested in [6], it can be employed to compress the high dimensional data and extract their significant features. PARAFAC2 is an extention to PARAFAC providing a more flexible decomposition by letting one dimension vary across the others [7].

The application of tensor decomposition can be significant for biomedical signals, such as EEG [8], where many transient events and movement related sources and artifacts are involved and most sources are inherently nonstationary. Moreover, the related brain neural process exhibit specific space-timefrequency locations. EEG signals in particular, consist of multichannel recordings with good temporal resolution which subsequently offers good time-frequency resolution. The application of tensor analysis is then logical and the data can be factorised into its space, time and frequency modes [9], [10], [11], [12]. Tensor factorisation has been also applied to multisubject data where the data can be factorised in the group level, identifying the common components [11], [13].

The coupling between neural processes has been investigated for various mental tasks such as attention, spatial navigation, perceptual binding and memory [14], [15], [16], [17]. The activity in different areas can be phase-coupled (i.e., display systematic phase-delays over trials), a phenomenon called phase-synchronization, which has been hypothesized to be an important mechanism for creating a flexible communication structure between brain regions. In the EEG field, the coupling between neural sources is usually measured by some metric of phase synchrony between electrodes. That is accomplished by obtaining the cross-spectrum from the frequency representation of the EEG via the Fourier transform. It is well-known that indexing phase-synchronization through scalp-sensor (scalpelectrode) measurements can be complicated by five problems (see also [18]): (i) the choice of reference electrode [19], [20], (ii) volume-conduction of source activity [21], (iii) the presence of noise sources [22], (iv) sample-size bias [22], and (v) the coupling between sensors may not be due to the activity closest to those electrodes [23], [22]. 
A variety of metrics has been proposed to assess EEG coupling with diverse levels of robustness to the issues above. For example, coherence [24] is the frequency domain equivalent of correlation in the time domain but it is heavily affected by the problems ii) and v) above. The phase slope index (PSI) [25] and phase locking value (PLV) [26] are extensions to coherence with the former attempting to find phase differences across frequencies and the latter tries to alleviate the amplitude correlations that confound coherence measurements of connectivity. In contrast, the imaginary part of coherence [14] was derived to alleviate volume conduction effects since instantaneous activations only have a real part. Similarly, the phase-lag index (PLI) computes the distribution of phase differences across observations by estimating the sign of the phase of individual observations [19], hence disregarding volume conduction effects. The pairwise phase consistency (PPC) [27] attemtps to alleviate the small sample size bias since its value is not affected by the number of observations but does not take into account volume conduction effects. Source estimation methods have also been attempted such as those based on a multivariate autoregressive model (MVAR) and independent component analysis (ICA) [28], [15]. Such methods attempt to project the scalp data into source components where volume conduction is factored out.

Tensor factorisation has been employed in brain connectivity studies primarily with the aim of dimensionality reduction or detection of dynamic changes [29], [30], [31], [32]. In this work, we extend the traditional tensor factorisation framework by considering complex valued tensors and we derive a connectivity metric based on the PLI [22] and the resulting factors. Complex tensors have been also used in factorisation schemes in [33], [34], [35]. In [33] a multilinear decomposition is performed that explicitly models phase shifts between trials while in [34] phase shifts are also considered between electrodes, a phenomenon which arises in electrocorticography data. Unlike those studies we do not only consider phase shifts but a general EEG model which requires complex trial activations. Subsequently, the proposed methodology enables the estimation of connectivity between the components of the decomposition. This is accomplished by the decomposition of the EEG into channel, frequency and complex trial components.

To this end, and to alleviate the problems ii. and iii. above we derive an EEG model in the complex domain and show that PARAFAC2 is more suitable than PARAFAC in factorising that model and extend the established metrics that estimate the coupling between sources. In section II, we describe the EEG model in the complex domain and together with an introduction on connectivity measures. Section III formulates the factorisation procedure and we demonstrate the suitability of PARAFAC2 and a connectivity metric that is defined on the extracted components. Section IV we expose the details of the benchmark dataset we use to evaluate our method and a real EEG dataset. In section $\mathrm{V}$ we show results on a benchmark connectivity dataset and real mild cognitive impairment (MCI) EEG data. Section VI puts the benchmark results into context, showcasing the conformity of the real $\mathrm{AD}$ data to prior pathological knowledge and the Scaffolding
Theory of Cognitive Aging [36]. Section VII concludes the paper.

\section{EEG CONNECTIVITY MODEL}

In this section we review the established model of EEG signals in the time and frequency domains. Based on that we expose the way that connectivity is modelled between a pair of brain sources based on AR processes. Furthermore, we briefly describe the general methodology behind connectivity metrics, highlight the fact that they operate on electrode space, and revisit the PLI [22] metric that is used in this paper.

\section{A. EEG measurement model}

The EEG measures the concurrent activity from multiple neural sources mixed into multiple sensors. Various forward models have been developed that map the way that such sources are propagate and are mixed into the scalp electrode sensors [37], [38]. Each source usually describes a separate mental process or group of related processes [8] and estimating their properties has been attempted in a variety of ways [39], [40], [12].

EEG is traditionally analysed with respect to channels and temporal samples resulting in a model:

$$
\mathbf{X}(t)=\mathbf{A S}(t)=\sum_{i=1}^{N} \mathbf{a}_{i} s_{i}(t)
$$

where $\mathbf{A} \in \mathbb{R}^{M \times N}$ describes the forward model of $N$ sources on $M$ electrodes and $\mathbf{S}(t) \in \mathbb{R}^{N}$ the source matrix where each source has a duration of $T$ temporal samples. EEG sources are commonly modelled as $k_{t h}$ order autoregressive (AR) processes [41]:

$$
s_{i}(t)=\sum_{\tau=1}^{r} h_{i}(\tau) s_{i}(t-\tau)+w_{i}(t)
$$

where $w_{i}(t)$ is white gaussian noise and $h_{i}(t)$ the AR process parameters for the $i_{t h}$ source. The parameters $h(t)$ can generate a variety of source types such as narrowband and lagged sources.

In the frequency domain the EEG model is written as:

$$
\mathbf{X}(f)=\mathbf{A S}(f)=\sum_{i=1}^{N} \mathbf{a}_{i} s_{i}(f)
$$

where $\mathbf{X}(f) \in \mathbb{C}^{M \times F}$ and $s_{i}(f) \in \mathbb{C}^{1 \times F}$ with $F$ the total number of frequency bins.

Due to the low signal-to-noise ratio of EEG signals, datasets usually consist of multiple measurements of the same state or task that generates the sources of interest. These measurements are called trials and aid in increasing the signal-to-noise-ratio (SNR) since undesired source activities are considered uncorrelated to the activities of interest. Similarly, in a continuous recording, data are segmented into non overlapping windows, again called trials, in order to facilitate analysis in shorter time segments. The model is then written as: 


$$
\mathbf{X}(f, k)=\mathbf{A} \mathbf{S}(f, k)=\sum_{i=1}^{N} \mathbf{a}_{i} s_{i}(f, k)
$$

where $s_{i}(f, k)$ is the complex source activation at the $f_{t h}$ frequency bin and $k_{t h}$ trial. The source model written in the frequency domain as:

$$
s_{i}(f, k)=h_{i}^{-1}(f) w_{i}(f, k)
$$

where $h_{i}^{-1}(f)$ denotes the inverse of $h(f)$ which is the fourier transform of the AR parameters. We can also write for simplicity:

$$
s_{i}(f, k)=p_{i}(f) y_{i}(f, k)
$$

where $p_{i}(f)$ and $y_{i}(f, k)$ now collect the terms corresponding to the frequency profile and the trial to trial variation respectively.

Such a model allows for time-shifts between trials and can be written as:

$$
\mathbf{X}(f, k)=\sum_{i=1}^{N} \mathbf{a}_{i} p_{i}(f) y_{i}(f, k)
$$

where $p_{i}(f) \in \mathbb{C}^{F}$ describes the complex amplitude of the $i_{t h}$ source at frequency $f$. The term $y_{i}(f, k)$ describes the trial to trial variations in amplitude and phase. Such a model allows for typical narrowband EEG sources such as alpha sources with sparse $p_{i}(f)$. It is important to notice that $y_{i}(f, k)$ is both a function of frequency and trial.

\section{B. EEG source connectivity}

When two sources are connected this can be modelled by bivariate AR processes such as [42]:

$\left[\begin{array}{c}s_{i}(t) \\ s_{j}(t)\end{array}\right]=\sum_{\tau=1}^{r}\left[\begin{array}{cc}h_{i}(\tau) & h_{i j}(\tau) \\ h_{j i}(\tau) & h_{j}(\tau)\end{array}\right]\left[\begin{array}{c}s_{i}(t-\tau) \\ s_{j}(t-\tau)\end{array}\right]+\left[\begin{array}{c}w_{i}(t) \\ w_{j}(t)\end{array}\right]$

The coefficients $h_{i j}$ describe the connection between the $i_{t h}$ and $j_{t h}$ sources also allowing for directionality in the connectivity when $h_{i j} \neq h_{j i}$.

Alternatively, each source may be considered in the frequency domain since many neural sources are oscillatory in nature [43]. The mixing model remains the same since frequency transforms are linear functions of the time domain signal. Note that the transformed sources $s_{i}(f)$ are now complex valued describing the power and phase as a function of frequency.

Coupled sources are then written as (see also Equations 5 and 6):

$$
\left[\begin{array}{l}
s_{i}(f) \\
s_{j}(f)
\end{array}\right]=\left[\begin{array}{cc}
p_{i}(f) & p_{i j}(f) \\
p_{j i}(f) & p_{j}(f)
\end{array}\right]\left[\begin{array}{l}
y_{i}(f) \\
y_{j}(f)
\end{array}\right]
$$

Coupled source appear when $p_{i j}(f) \neq 0$ and contain mixed effects such as:

$$
s_{i}(f)=p_{i}(f) y_{i}(f)+p_{i j}(f) y_{j}(f)
$$

where $p_{i j}$ describes the coupling between sources $i$ and $j$.

\section{Connectivity metrics}

Consider two electrodes $x_{i}$ and $x_{j}$, the coupling between them can be estimated by a variety of methods which use the cross-spectrum as an initial step:

$$
F_{i, j}(f, k)=x_{i}(f, k) x_{j}(f, k)^{*}
$$

which is the cross-spectrum between the electrodes at frequency $f$ and trial $k$. Subsequently, coupling is assumed to exist if an electrode is leading (or lagging) in phase consistently over trials. Several measures have been developed one of which is the PLI:

$$
\Psi_{i j}(f)=\left|\mathbb{E}\left\{\operatorname{sign}\left(\Im\left(x_{i}(f) x_{j}(f)^{*}\right)\right)\right\}\right|
$$

where the expectation is taken over trials. If the electrode signals are of consistently different phase then $\Im\left(x_{1}(f) x_{2}(f)^{*}\right)$ will be of the same sign and $\Psi(f)$ will tend to 1 , otherwise it will be around 0 . Measuring the brain connectivity using the PLI is optimal only for a pair of coupled sources as shown in [22]. For more than 2 coupled sources, cross-source interference arises and the optimality of the measure is lost in terms of volume conduction accuracy of spatial location. The PLI was chosen as the metric of interest since it is widely used in neuroimaging studies [44], [19], [45] and is suitable for estimating phase differences between components. In this work our primary focus is not the optimal choice of metric but the extension of metrics to be used through tensor decomposition.

\section{COMPLEX TENSOR FACTORISATION}

In this section we define the tensor model that is used in this paper and we show that the PARAFAC2 [2], [3], [7], [46] tensor formulation conforms to the EEG model described in the previous section. Next, we show that decomposing the EEG signal into its constituent components through the PARAFAC2 algorithm allows for estimation of the connectivity between the estimated components and not in electrode space anymore. In that light, we expand upon the PLI and propose a combined spatial-spectral-connectivity metric that is only available through tensor factorisation.

\section{A. Tensor model}

In order to alleviate the aforementioned issues with simple connectivity measures we propose a tensor factorisation procedure where the EEG is decomposed into distinct components in the complex domain. We consider a tensor EEG model, directly resulting from Equation 7, as a sum of $N$ components where each one is described by a triplet of real activations over electrodes a, over complex frequencies $\mathbf{p}$, and complex trials y as:

$$
\begin{aligned}
& \mathbf{X}(f)=\mathbf{A} \mathbf{P}(f) \mathbf{Y}(f) \\
& =\mathbf{A}\left[\begin{array}{lll}
p_{1}(f) & & \\
& \ddots & \\
& & p_{N}(f)
\end{array}\right]\left[\begin{array}{lll}
\mathbf{y}_{1}(f) & \ldots & \mathbf{y}_{N}(f)
\end{array}\right]
\end{aligned}
$$


where $\mathbf{X}(f) \in \mathbb{C}^{M \times K} \cdot \mathbf{y}_{i}(f) \in \mathbb{C}^{K \times 1}$ are the complex activations of the $i_{t h}$ component over $K$ trials placed in the matrix Y. $\mathbf{P} \in \mathbb{C}^{N \times N}$ is a diagonal matrix holding the complex frequency profiles of the $N$ components in the diagonal for a single frequency $f$. This model is the PARAFAC2 model [7]. If the source activations were identical for all frequencies i.e. $s_{i}\left(f_{i}\right)=s_{i}\left(f_{j}\right)$ then the model would correspond to the typical and simpler PARAFAC model with:

$$
\begin{aligned}
& \mathbf{X}(f)=\mathbf{A P}(f) \mathbf{Y} \\
& =\mathbf{A}\left[\begin{array}{lll}
p_{1}(f) & & \\
& \ddots & \\
& & p_{N}(f)
\end{array}\right]\left[\begin{array}{lll}
\mathbf{y}_{1} & \cdots & \mathbf{y}_{N}
\end{array}\right]
\end{aligned}
$$

The main difference between the two models being that $\mathbf{Y}$ is the same for all frequencies in PARAFAC while PARAFAC2 adds the flexibility for variable $\mathbf{Y}(f)$. Also, the $\mathbf{Y}(f)$ can potentially have different dimensions for each $f$ in PARAFAC2. Lastly, both PARAFAC and PARAFAC2 provide unique decompositions but whilst in PARAFAC the uniqueness is through the strict trilinearity of the data, for PARAFAC2 and additional constraint needs to be added (see Section III-C and [7], [46]).

There are two reasons that the source activations are dependent on the frequency: a) due to $w_{i}(f, k)$ being a complex source amplitude it achieves different complex values for different frequencies and trials, and b) differences between trials can be accommodated by considering that a variable process such as $w_{i}^{\prime}(f, k)=d_{i}(f, k) w_{i}(f, k)$. For phase-shifts between trials we can consider: $d_{i}(f, k)=\exp \left(-j \theta_{k} f\right)$ being also a function of frequency and trials. Hence, this formulation allows the decomposition of EEG into complex sources that are potentially not phase locked to a stimulus.

\section{B. Connectivity estimation in component space}

It then becomes possible to measure connectivity information in component space by considering the trial activations. The connectivity measure is performed similarly to the crossspectrum between electrodes but now is between components:

$$
\Psi_{i j}^{s}(f)=\mid \mathbb{E}\left\{\operatorname{sign}\left(\Im\left(\mathbf{s}_{i}(f) \mathbf{s}_{j}(f)^{*}\right)\right\} \mid\right.
$$

which measures the coupling between component $i$ and $j$ where $\mathbf{s}_{i}(f)=p_{i}(f) \mathbf{y}_{i}(f)$. In order to obtain a connectivity profile over the scalp we can use that PLI value weight by the activation of the sources over the scalp:

$$
\mathbf{C}_{i j}^{a}=\Psi_{i j}^{s}\left(\mathbf{a}_{i} \mathbf{a}_{j}^{T}+\mathbf{a}_{j} \mathbf{a}_{i}^{T}\right)
$$

which gives a symmetric connectivity profile of the coupling between the two sources. Furthermore, the frequency information can be incorporated by weighting by the mean frequency magnitude over a specific band of the desired sources:

$$
\mathbf{C}_{i j}^{a f}=\Psi_{i j}^{s}\left(\mathbf{a}_{i} \mathbf{a}_{j}^{T}+\mathbf{a}_{j} \mathbf{a}_{i}^{T}\right)\left(\frac{1}{L} \sum_{l}^{L}\left|\mathbf{s}_{i}\left(f_{l}\right)\right|+\left|\mathbf{s}_{j}\left(f_{l}\right)\right|\right)
$$

with $f_{l}$ corresponding to the range of frequencies that are considered.

\section{Complex PARAFAC2}

The factorisation procedure entails alternating least squares minimisations of the following equation:

$$
\underset{\mathbf{A}, \mathbf{P}(f), \mathbf{Y}(f)}{\operatorname{argmin}}\|\mathbf{X}(f)-\mathbf{A P}(f) \mathbf{Y}(f)\|^{2}
$$

However, the solution to the factorisation of equation 18 is not unique [7] and the PARAFAC2 model imposes an additional constraint to facilitate uniqueness. This is accomplished by having the cross-products of the $3^{r d}$ mode be constant over its index:

$$
\mathbf{Y}\left(f_{i}\right)^{H} \mathbf{Y}\left(f_{i}\right)=\mathbf{K}, \forall i
$$

which is implemented by having $\mathbf{Y}(f)=\mathbf{Q}(f) \mathbf{H}$ where $\mathbf{Q}(f) \in \mathbb{C}^{K \times N}$ is an orthonormal matrix and $\mathbf{H} \in \mathbb{C}^{N \times N}$ any square matrix. That leads to the modified factorisation algorithm as:

$$
\underset{\mathbf{A}, \mathbf{P}(f), \mathbf{H}}{\operatorname{argmin}}\left\|\mathbf{Q}(f)^{H} \mathbf{X}(f)-\mathbf{A} \mathbf{P}(f) \mathbf{H}\right\|^{2}
$$

which is solved by standard PARAFAC algorithms. Note that the minimisation over $\mathbf{A}$ is performed in the real domain.

The constraint is not optimal for EEG data since it enforces the complex inner product between sources to be equal for all frequencies which is not necessarily true. However, in our case where sources are coupled only in a narrow band (e.g. alpha sources), the inner product in the rest of the frequency range will be weighted by the activity on the 2 nd mode. The results of the simulation also support the usefulness of the constraint in our setting (c.f. V-A). In Algorithm 1 we provide a pseudocode for the proposed algorithm while in Figure 1 we provide a block diagram of the different steps.

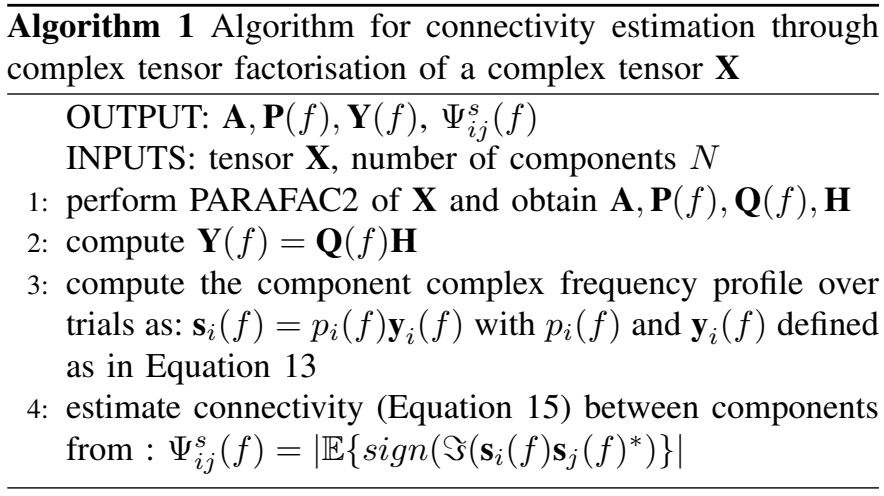

\section{EXPERIMENTAL VALIDATION AND APPLICATION TO REAL WORLD SIGNALS}

\section{A. Validation with synthetic data}

The PARAFAC2, PARAFAC and MVAR-ICA algorithms are evaluated initially on the Berlin Brain Connectivity Benchmark [42]. This framework provides the capability of EEG data generation that resemble as much as possible real EEG data with customisable parameters. The simulation includes a realistic a 108-channel real head model with 2000 leadfield vectors where each one belongs to one of the 8 regions of interest of the human brain [RAI, RAS, RPI, RPS, LAS, LPI, LPS] with R: right, L:left, A: anterior, P: posterior, S: 


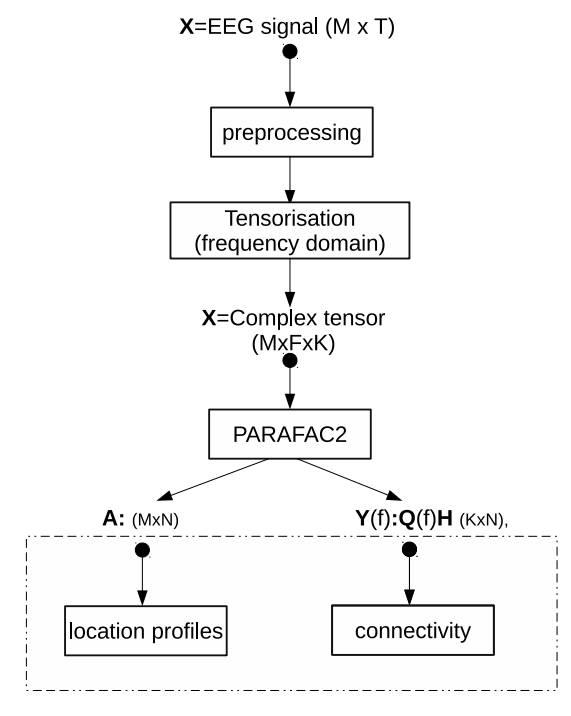

Fig. 1. Block diagram of the proposed method highlighting the main steps of the algorithm.

superior, I: inferior. The framework also generates pseudoEEG sources that can be either narrowband (e.g. alpha band) or pink noise sources corresponding to background brain activity. The generated sources are centered around a lead-field vector and then spread within the same ROI according to a Gaussian distribution with $\sigma$ in the range of $[10-40] \mathrm{Hz}$. The framework also enables the creation of coupled sources based on the bivariate AR model of Equation 8 or not coupled sources with zeros on the off-diagonals. The default settings provided in the framework correspond to a realistic EEG recording [42]. These include a pair of coupled sources with spread over the cortex resembling a brain network and a large number of background EEG activities. The framework can generate multiple datasets where each one contain a different randomized generation of all the sources, locations and noise.

Each dataset is sliced into 1 second trials resulting in a EEG of dimensions $108 \times 180$. A common average reference is applied and the data are also detrended and baselined. Furthermore, we tensorise the data with the short time fourier transform with $1 \mathrm{~Hz}$ Hanning tapers in the range of $[1-40] \mathrm{Hz}$.

This results in an EEG of dimensions $108 \times 40 \times 180$ which is input to the PARAFAC2, PARAFAC algorithms (1). For the PARAFAC2 algorithm, in order to avoid the effect of local minima we perform 10 runs of 10 initialisations each and retain the run with the highest coupling value. This is performed for both the datasets that contain coupled sources and the ones that do not. Both tensor factorisation algorithms provide a matrix of spatial activations $\mathbf{A} \in \mathcal{R}^{M \times N}$ which contains the spatial topogrophy of the $N$ components. Also, they provide $\mathbf{Y}(f)$ which is used in Equation 15 in order to estimate the coupling strength between the $N$ components.

For the comparison to MVAR-ICA we use the 2D EEG after spectral filtering. The MVAR-ICA method initially fits an MVAR model in order to obtain the coupling parameters between electrodes $(\mathbf{B}(\tau))$. Then, ICA is performed on the residuals of that process in order to obtain the MVAR parame- ters of the independent components $\left(\mathbf{B}_{i c a}(\tau)=\mathbf{W B}(\tau)\right)$ where $\mathbf{W}$ is the unmixing matrix from ICA. The MVAR parameters are used then for connectivity estimation and the leadfield matrix $\mathbf{H}=\mathbf{W}^{-1}$ for getting the locations of the sources. For MVAR-ICA we set $N=108$ since it obtained the higest performance.

For the purposes of this study we desire to evaluate: a) the performance of an algorithm in estimating the coupled sources in terms of the power ratio (PR) of the coupled sources to the whole EEG power and $b$ ) the influence of the number of components of the tensor factorisation to the performance of the algorithm. Note that the concept is inherent to the framework and is different from the generally accepted SNR. The relation is $P R=\frac{S}{S+N}$ whereras $S N R=\frac{S}{N}$.

We define three different metrics to accomplish this goal. Firstly, the explained variance (EV) of the tensor factorisation which describes how well the algorithm is fit to the data (ranges from 0 to 1 ):

$$
E V=\frac{\sum_{f}\|\mathbf{A P}(f) \mathbf{Y}(f)\|_{F}^{2}}{\sum_{f}\|\mathbf{X}(f)\|_{F}^{2}}
$$

Secondly, a connectivity estimation metric CONN as described in [42] which describes the ability of the algorithm to detect whether there exist coupled sources in an EEG segment or recording. A correct estimation is awarded with +1 while a wrong one with -2 with the chance level being at -0.5 . Lastly, the LOC metric as described in [42] which estimates how well the algorithm can identify the coupled sources and their spatial profile in terms of the brain octant they belong to. The LOC metric awards for each source +0.5 when the octant is predicted correctly and -0.5 otherwise. Chance level of this metric is -0.5 .

In summary the settings of the simulated EEG are:

- Channels: 108

- Sampling frequency: $100 \mathrm{~Hz}$

- Coupled sources: 2

- Coupled source locations: random in ROIs [RAI, RAS, RPI, RPS, LAS, LPI, LPS]

- Noise sources: 500

- AR model order: 5

- Power ratio (PR) range: 10\% - $90 \%$

- Sensor noise: $10 \%$

- Chance a dataset contains coupled sources: $50 \%$

- Datasets for each PR: 250

- Duration of each dataset: 3 minutes

- Realistic head model lead-fields: 2000

a) CONN and LOC computation: For all three methods (PARAFAC2, PARAFAC, MVAR-ICA) we obtained firstly the coupling strength for each pair of components in the decomposition. The pair with the highest coupling was selected for each dataset and PR:

$$
C R(i, P R)=\max \left(\mathbf{C}_{i, P R}\right)
$$

where $\mathbf{C}_{i, P R}$ is an $N \times N$ matrix containing the coupling strenghts between the components for dataset $i$ and some PR. The CONN metric was estimated by finding the threshold 
that maximally separated the coupling strength between the datasets that contained coupled sources and the ones that did not. Then it was estimated by averaging over the correct $(+1)$ and wrong (-2) estimations over each dataset.

The LOC was estimated by correlating the obtained spatial component of each of the paired sources with the highest coupling strength with each of the lead-field of the head model and picking the octant with the highest correlation. The LOC was estimated for the datasets that contained coupled sources using the scoring rule above with a $(+1)$ if the coupled source was identified at the correct octant and (-2) otherwise.

\section{B. Illustration in $\mathrm{MCI}$}

The algorithm was applied on two electroencephalography (EEG) datasets on a memory task (VSTMBT) from MCI patients and control subjects [47]. Both samples of MCI patients presented with the typical pattern of VSTMB impairments reported in previous studies involving patients with Alzheimers disease i.e., larger performance drop of memory binding than of memory for shape only (see [47] for full behavioural data). In dataset-1, there were 128-channel recordings from 13 patients with mild cognitive impairment (MCI) and 14 control subjects (mean age: 73) while for dataset-2 there were 64channel recordings from 10 patients with MCI and carrying the gene of familial Alzheimer's disease (FAD) and 10 control subjects (mean age: 44). Similar preprocessing is performed in the EEG signals of the AD datasets resulting in tensors $128 / 64 \times 40 \times 50$ with the number of trials slightly different between subjects with identical algorithm parameters as in the benchmark dataset.

a) The Visual Short-Term Memory Binding Test (VSTMBT): The VSTMBT required participants to remember visual arrays in which three black shapes (Shape Only condition) or coloured shapes (Shape-Colour Binding Condition) were presented for 2 seconds. After a brief delay (1 second), a test display appeared showing the same or different items all presented in new random locations. The task was to indicate whether the study and test display showed the same $(50 \%$ of the trials) or different items. Different trials in the Shape Only condition presented two new shapes at test. Different trials in the Shape-Colour Binding condition presented two re-arranged combinations of shape and colour (i.e., two shapes swapped their colours at test). Each condition presented 100 trials in random order. Conditions were counterbalanced across participants (see [48] for a detailed description of the task).

\section{RESUlts}

\section{A. Simulated EEG}

Firstly, we apply the complex tensor factorisation algorithm for a range of PR values and for two cases for the number of components $N=2$ and $N=8$. The number of components was set to a maximum of 8 to avoid non-uniquness issues. For each PR value we generate 250 datasets where in each one the signal sources are in the alpha frequency band and the noise sources are pink noise realisations. Then, we compute the three metrics and the results are seen in Figure 2. We also show the performance of the PARAFAC and MVAR-ICA methods [28]. For MVAR-ICA the number of components was set to $N=108$ since that obtained the higest performance.

In Figure 3 we show the effect on the metrics of varying connection strength between the coupled sources. In Figures 4 and 5 we show the effect on the metrics of the spread of the coupled sources over the cortex and their frequency band respectively. For these simulations we set the $P R$ to 0.5 and $N=8$. These simulations were performed to obtain a more qualitative estimation of the algorithm's properties as suggested in [49].

\section{B. EEG from samples at risk of Alzheimers dementia}

For both datasets, we applied a $N=8$ component complex PARAFAC2 algorithm and calculated the connectivity map on the pair of sources with the highest connection value on the alpha $(8-12 \mathrm{~Hz})$, beta $(13-20 \mathrm{~Hz})$ and theta $(4-7 \mathrm{~Hz})$ frequency bands, see equation 17. The choice of components was based on finding a point when the explained variance reached a ceiling level, Figure 6, and similarly for the consistency of the PARAFAC2 estimation, Figure 7. Consistency is defined as the between subject average standard deviation of the obtained values for the highest coupled pair of components. In Figures 8,9 and 10 we show the connectivity map obtained with tensor factorisation on the alpha, beta and theta bands. Figure 11 shows the connectivity map obtained through the standard PLI measure for beta bands only. An example source pair is shown in Figure 12.

We also calculated the power ratio (PR) similar to the benchmark dataset. The differences in PR values between groups, task and frequency band were not significantly different with a mean of 0.35 for the MCI and 0.32 for the FAD group.

There were statistically significant differences with an unpaired $t$-test in the coupling metric, Equation 15, between the MCI group and the matched controls $(p<0.05)$ for both the binding and the shape tasks with the MCI group exhibiting greater coupling. No differences were found for the MCI-FAD group and the matched controls. In terms of the power weighted metric of Equation 17 we performed a 3-way ANOVA analysis separately for the MCI and MCIFAD datasets. The factors were condition (patient-control), task (binding-shape) and frequency band (alpha-beta-theta), with the dependent variable being the average strength over the whole scalp. Significant differences were found in condition and frequency band for both datasets (MCI/condition: $F=8.49, p<0.01$, MCI/band: $F=3.77, p<0.05$, MCI-FAD/condition: $F=5.24, p<0.05$, MCI-FAD/band: $F=5.11, p<0.01$ ) with significant differences in task for only the MCI dataset only (MCI/task: $F=6.24, p<0.05$ ). Interactions between task and condition revealed significant differences in the MCI group between the controls and patients for both tasks. We also tested the correlation between the coupling metric and power weighted metric with significant anti-correlation $(r=-0.5, p<0.01)$ for the shape task and the patient group for both datasets. 

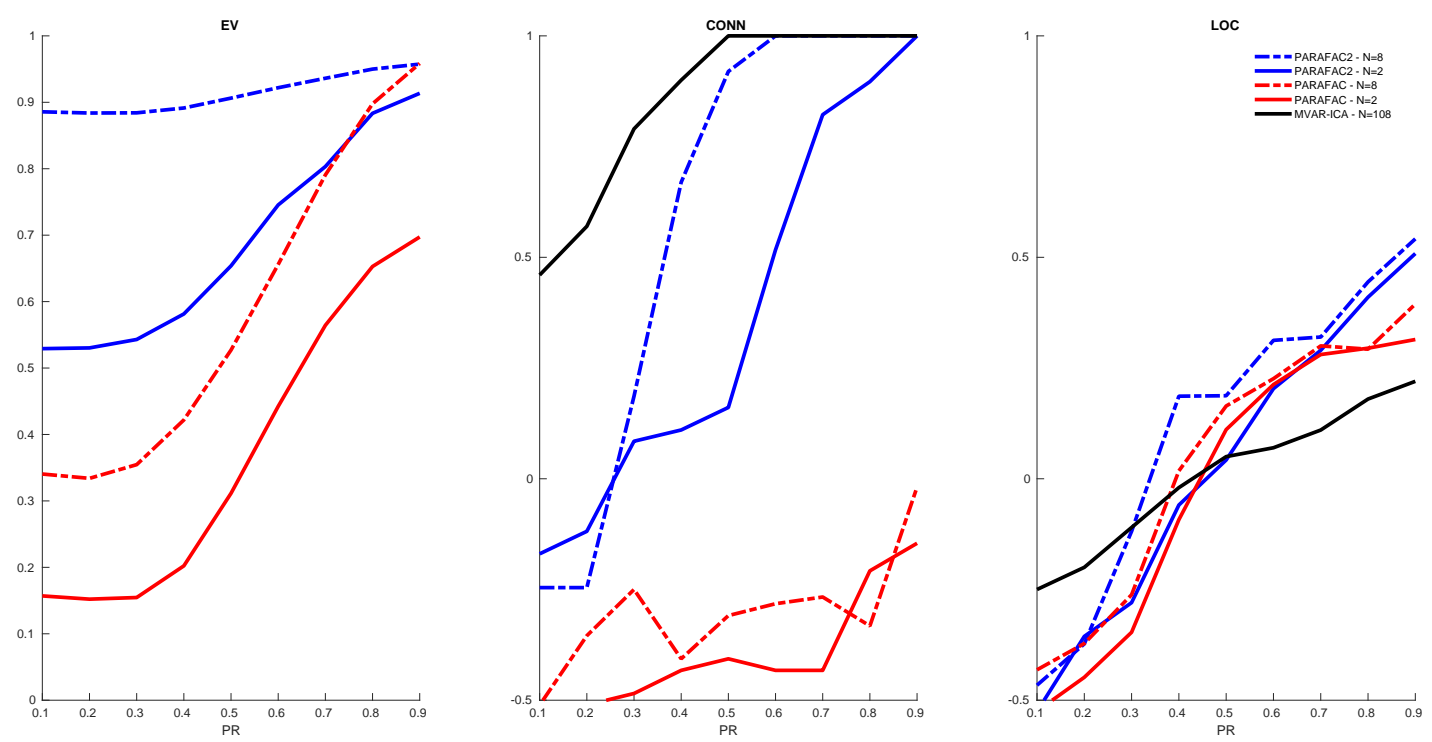

Fig. 2. Explained variance (EV), CONN and LOC of the PARAFAC2, PARAFAC and MVAR-ICA algorithms for a range of PR values and different number of components. For each PR value we generate 250 datasets where in each one the signal sources are in the alpha frequency band and the noise sources are pink noise realisations. The MVAR-ICA needs the max number of components $(N=108)$ to reach the best performance.
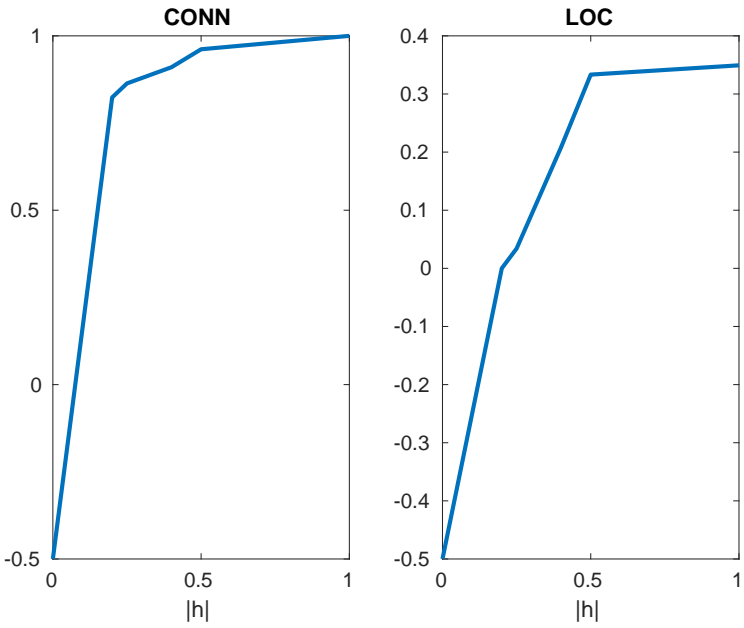

Fig. 3. CONN and LOC of the PARAFAC2 algorithm for a range of connection strenghts between the coupled sourrces. The PR values are set to 0.5 and $N=8$. For each value of the connection strength, expressed as the norm of the off-diagonal of Equation 9

\section{DISCUSSION}

Complex tensor factorisation enables the estimation of brain connectivity in scalp source space and its efficacy was shown in both a benchmark EEG dataset and two EEG datasets comprising of MCI patient and control data. Performing tensor factorisation in the complex domain enables the calculation of brain connectivity metrics since we obtain estimates of the sources phase information. The theoretical justification of using PARAFAC2 as the tensor factorisation model was demonstrated in sections II and III. In the former we describe the way neural processes are described in the complex domain
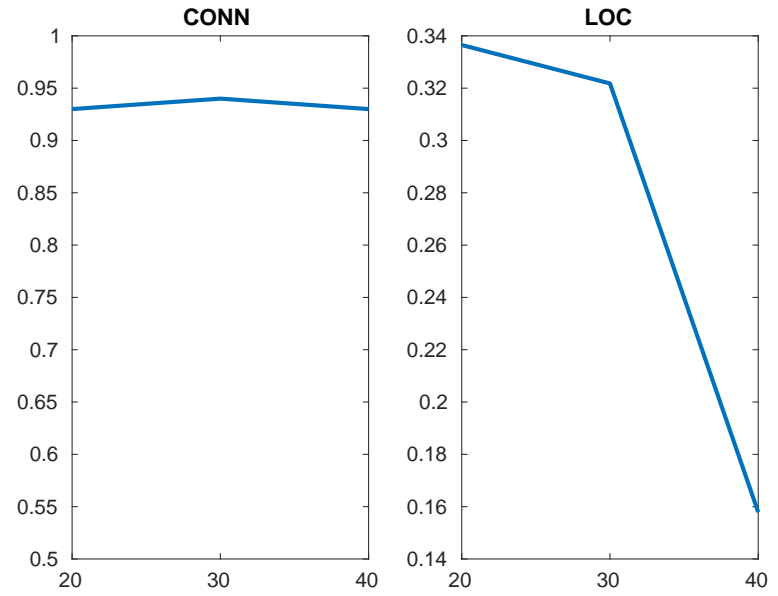

Fig. 4. CONN and LOC of the PARAFAC2 algorithm for a range of source location spreading parameter between the coupled sources. The PR values are set to 0.5 and $N=8$.

and show their dependency on frequency and trial for both phase- and non phase-locked sources.

Initially, we measured the performance of the algorithm in the benchmark dataset. Complex PARAFAC2 algorithm is better than complex PARAFAC in decomposing the underlying data into components that reflect the true activity. Also, PARAFAC2 exhibits good detection performance, see Figure 2. For all but low PR values $(<0.3)$ PARAFAC2 also outpeformed MVAR-ICA. Using such a benchmark dataset we were able to generate EEG signals of varying power ratios and evaluate the performance for a wide range. We measure three different performance metrics describing various aspects of the algorithm. In Figure 2 we showed the perfomance 

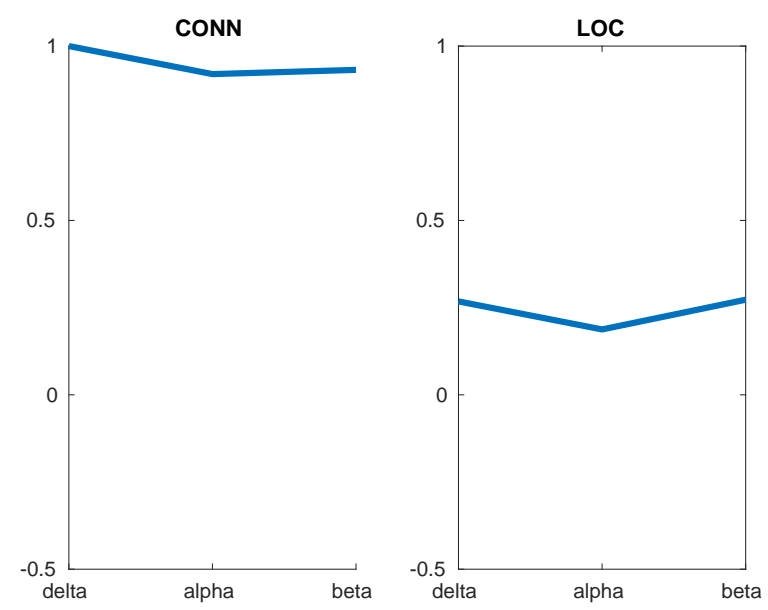

Fig. 5. CONN and LOC of the PARAFAC2 algorithm for different frequency bands of the coupled sources. The PR values are set to 0.5 and $N=8$.

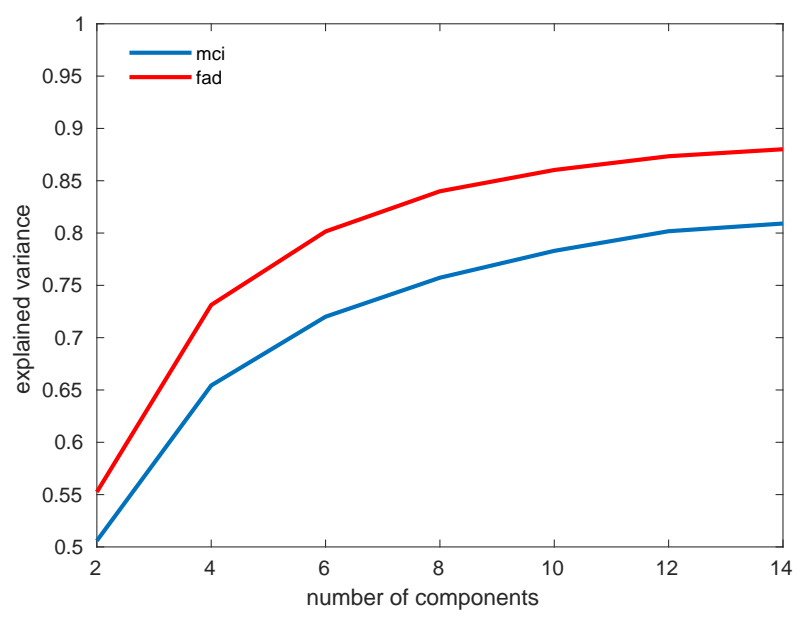

Fig. 6. Explained variance for the MCI and FAD datasets for an increasing number of components. It can be observed that a ceiling level is reached at $N=8$ for both datasets.

in terms of explained variance for various PR values and number of components $N$. This value describes how well the PARAFAC2 model can fit the data. Moreover, the efficacy in terms of extracting the desired sources is estimated in two ways. Firstly, by computing the CONN metric. This metric is an objective measure of how powerful the signal has to be in order to reliably extract its phase information. As observed, increasing the PR increases the CONN for PARAFAC2 but not for PARAFAC. MVAR-ICA outpeformed PARAFAC2 for very low PR $(<0.4)$ in terms of the CONN metric. Secondly, we estimate whether the location of the highest connected sources correspond to their true location. For all cases the increased efficacy with increased PR and $N$ was evident with PARAFAC2 outpeforming PARAFAC and MVAR-ICA. Further increase in $N$ improves the results but the uniqueness requirements are violated as obtained through empirical results for PARAFAC2 [7], [50].

MVAR-ICA seems better at lower PRs for connectivity

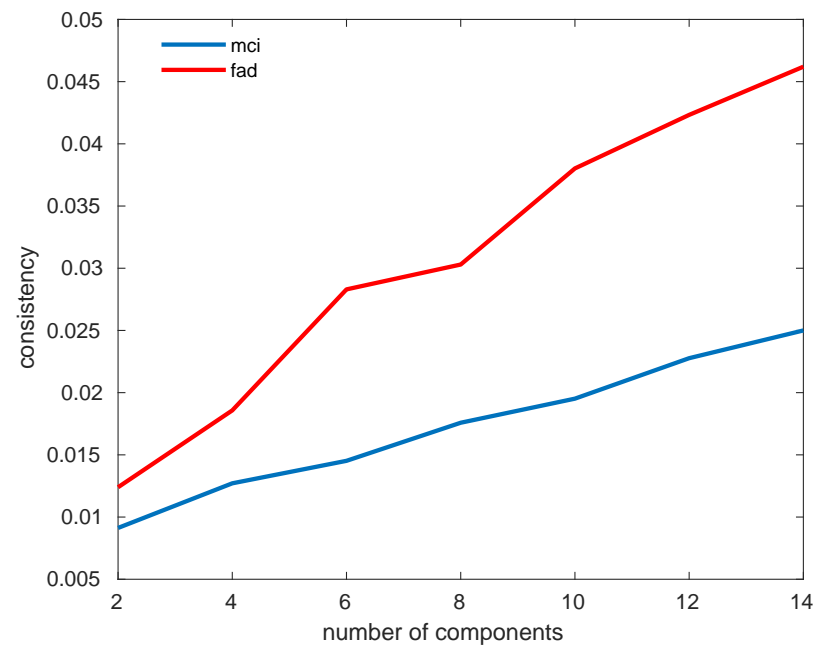

Fig. 7. Consistency of successive runs of the algorithm for the MCI and FAD datasets for an increasing number of components. Consistency is defined as the between subject average standard deviation of the obtained PLI values for the highest coupled pair of components.

measurement but suffers in localising the sources. The reasons for that are that the data conform exactly to the MVAR model while PARAFAC2 can deal with other data types such as data that are phase correlated only. Furthermore, PARAFAC2 being a tensor based method allows for inspection of the frequency and trial-to-trial variations separately, a functionality that is not possible with MVAR-ICA. For example, only with PARAFAC2 one can inspect the phase information for a particular time window and frequency band. Lastly, PARAFAC2 estimates $N *(M+F * K+K)$ parameters while MVARICA needs to estimate $\left(M^{2} * p\right)$ parameters for the MVAR step where $p$ is the model order and $N * M$ for the ICA step. In terms of this dataset MVAR-ICA needs almost 4-fold increase in the number of parameters to be estimated, something that is evident because the number of parameters vary with $M^{2}$. Therefore the PARAFAC2 model is more parsimonious than MVAR-ICA while providing better analysis capabilities.

The obtained values for CONN and LOC can also be contrasted to those obtained in [42] where $\mathrm{CONN}=0.51$ and $\mathrm{LOC}=0.5$ over 100 datasets for PR in the range of [0.20.9]. However, their work assumes prior knowledge of the oscillatory sources' frequency bands; such information is not required in our algorithm. Furthermore, based on that prior knowledge of the frequency band a decision for the CONN or LOC was made in [42] only when the PR is $50 \%$ higher than the background activity. Essentially, this renders the simulations for $P R \geq 0.7$. In this work, we aim to describe the performance of an algorithm without any heuristic measures and establish a kind of benchmark that future studies can be compared to.

In order to obtain a more qualitative idea of the capability of PARAFAC2 we performed the simulations shown in Figures 3,4 and 5 as suggested in [49]. Regarding connection strength we can see that PARAFAC2 is invariant until up to very low connection strengths (0.2) for the CONN and about 0.5 for the 


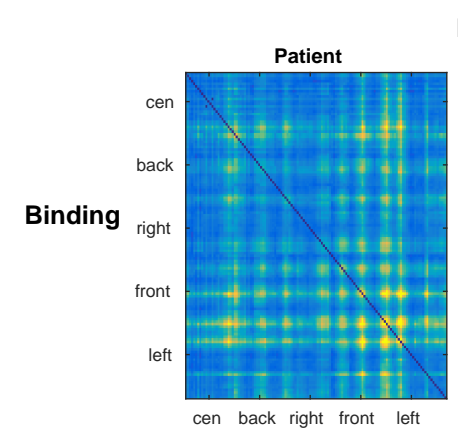

$\mathrm{MCl}$
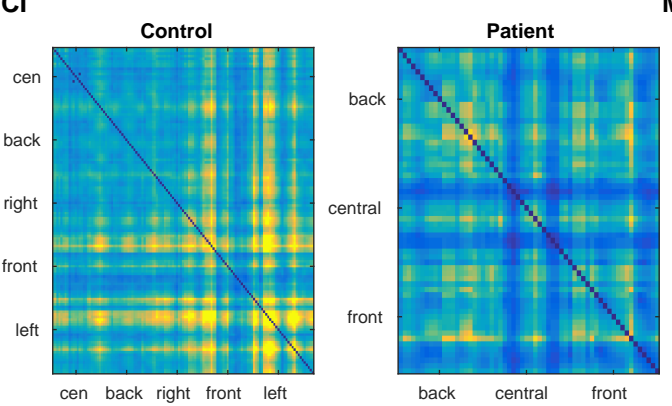

MCI-FAD
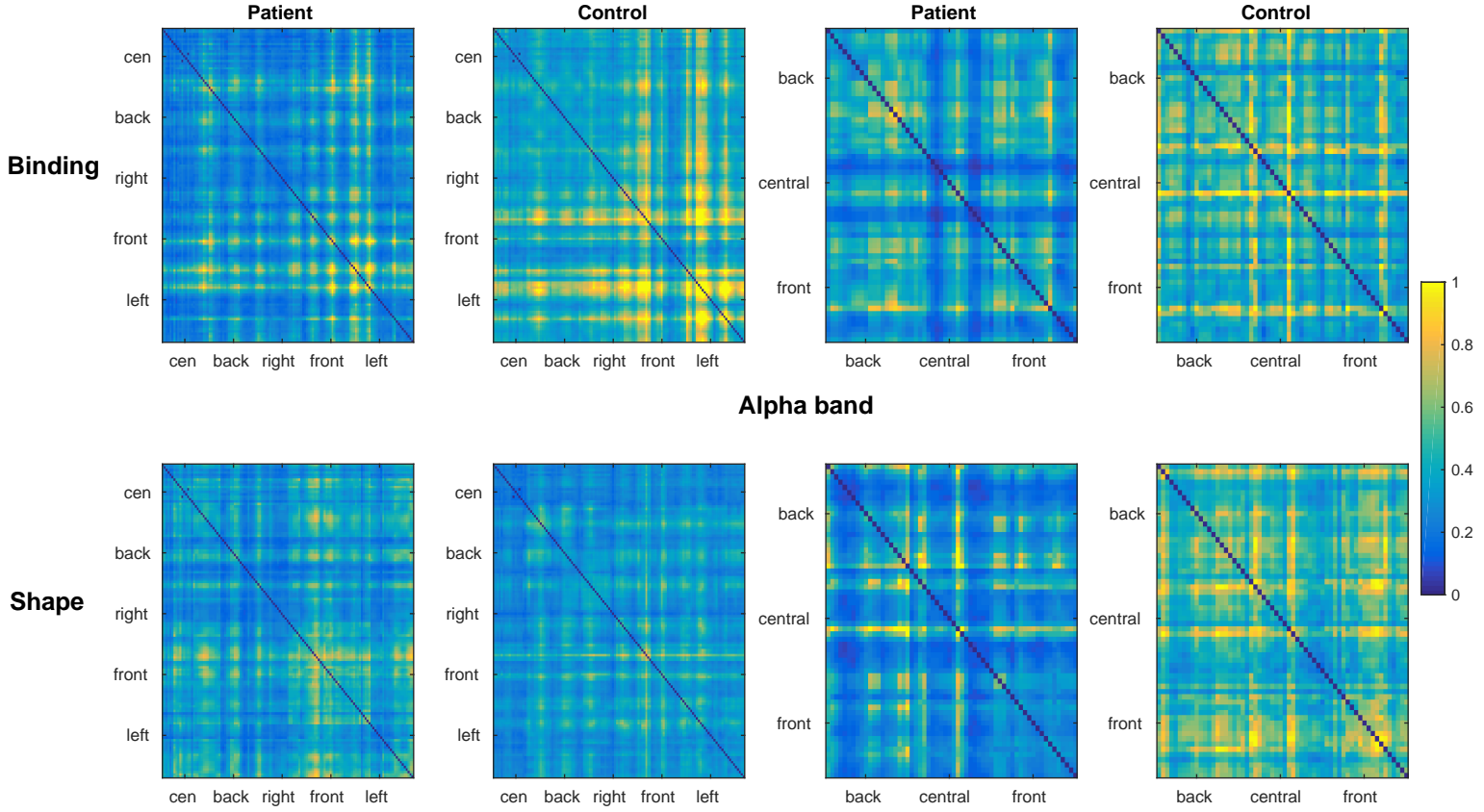

Fig. 8. Connectivity map for the tensor-PLI measure and the MCI (left) and MCI-FAD (right) datasets in the alpha band for $N=8$. Each point indicates the coupling between two electrodes which have been grouped into five regions over the scalp.

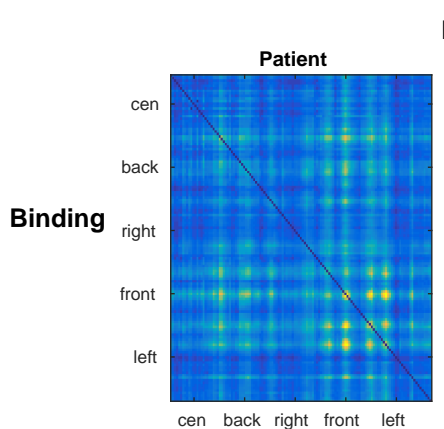

MCI
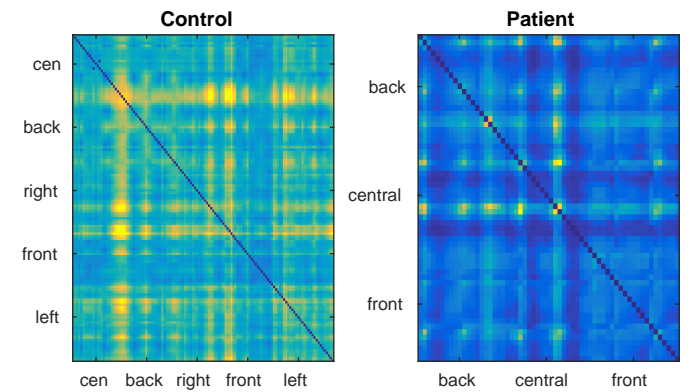

\section{Beta band}
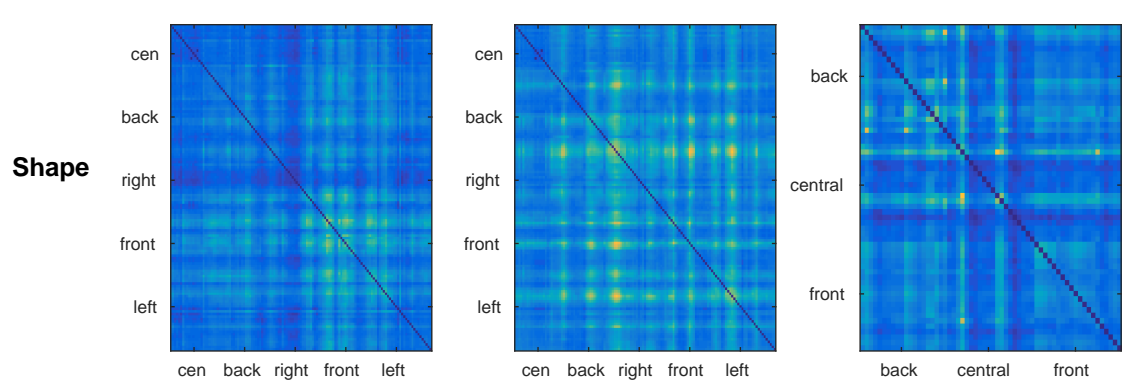

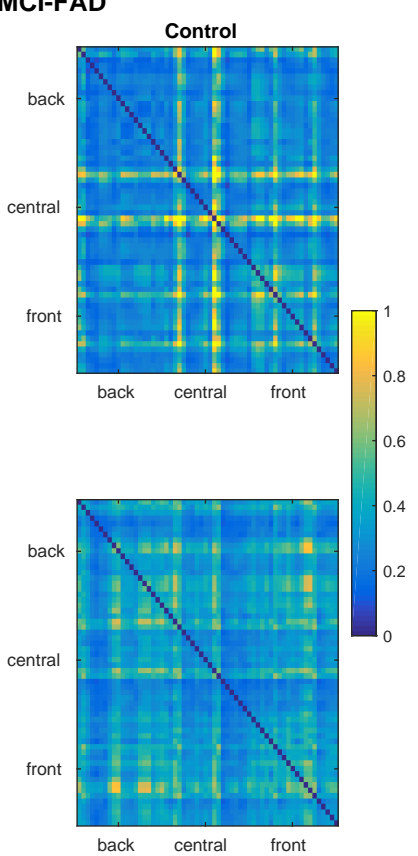

Fig. 9. Connectivity map for the tensor-PLI measure and the MCI (left) and MCI-FAD (right) datasets in the beta band for $N=8$. Each point indicates the coupling between two electrodes which have been grouped into five regions over the scalp. 

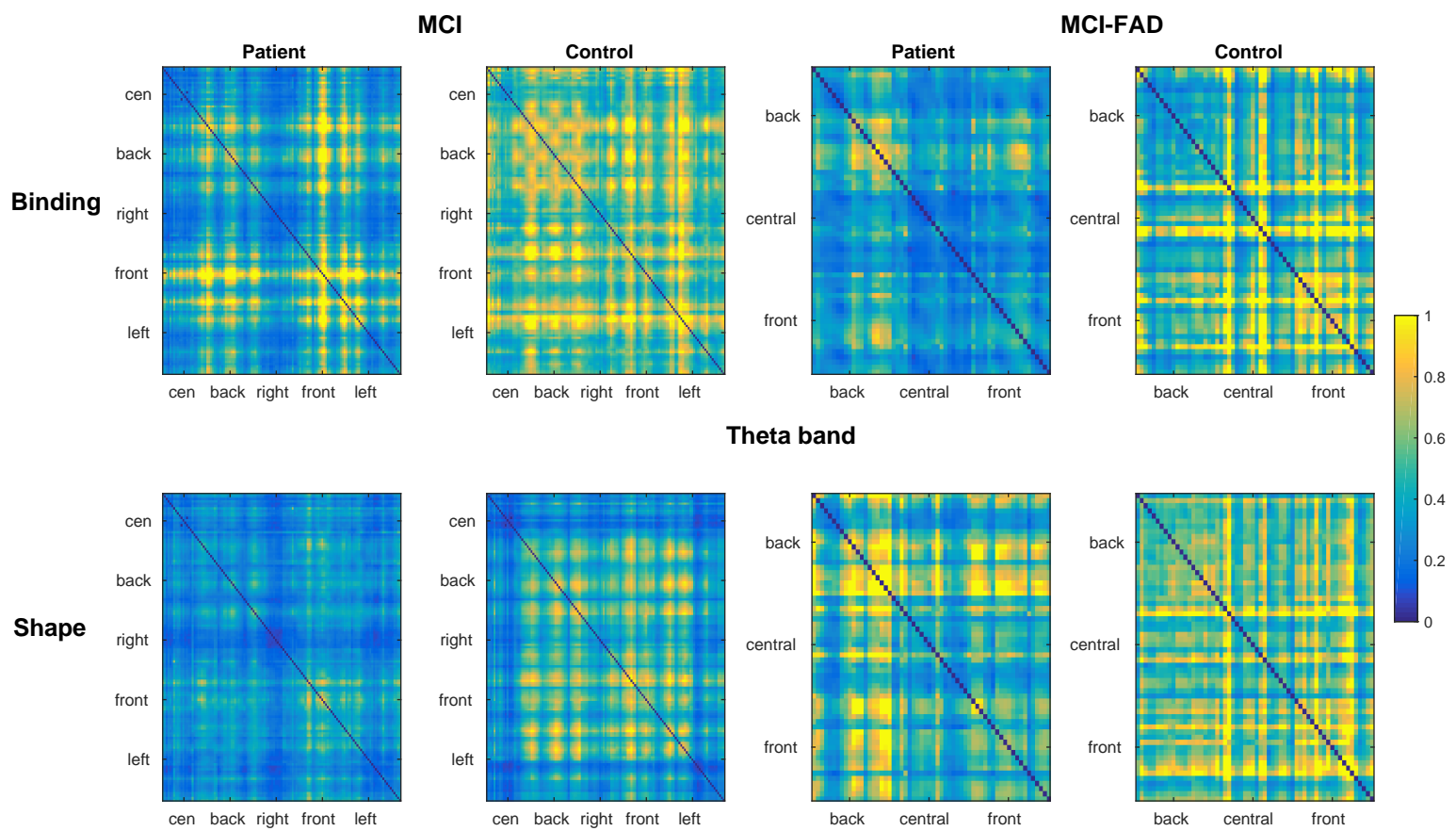

\section{Theta band}
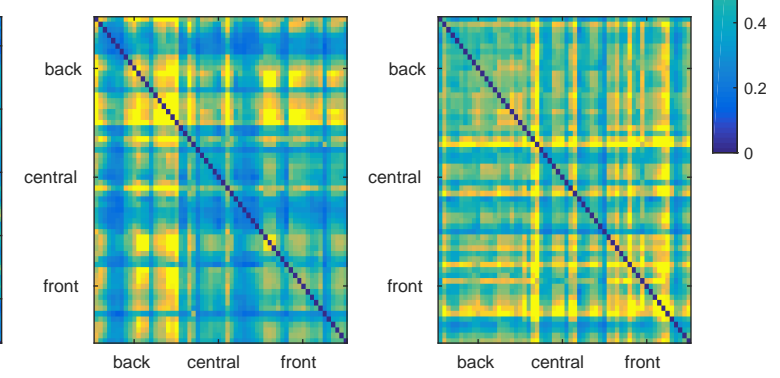

Fig. 10. Connectivity map for the tensor-PLI measure and the MCI (left) and MCI-FAD (right) datasets in the theta band for $N=8$. Each point indicates the coupling between two electrodes which have been grouped into five regions over the scalp.

LOC measure. In terms of the distributed or localised nature of the sources the only downgrade in performance was for high spread as seen in Figure 4 and the LOC measure. This is because when the sources are spread they contain many lead-field components and it gets harder to identify the correct octant. Finally, PARAFAC2 was invariant to the frequency band as seen in Figure 5.

In the real EEG dataset we demonstrate the performance of the algorithm by applying to two task based EEG datasets. The choice of the number of components, $N=8$, was performed on the basis of diminishing returns on the explained variance and the gradual decrease of consistency between different runs of the PARAFAC2 algorithm, see Figures 6 and 7. We avoided the use of the CONCORDIA [1] as its theoretical properties for complex PARAFAC2 are not known in the literature. The motivation behind the tests is to test if prior physiological knowledge about the Alzheimer's patients can be also obtained through complex PARAFAC2. Based on a memory task [47] the expectation is that the reduced performance of the patient groups should be explained by the loss of neural mechanisms. As seen in Figures 8, 9 and 10, the power adjusted connectivity metric, as defined in Equation 17, of the control groups is higher for both tasks and for the alpha, beta and theta frequency bands. In contrast, the synchronisation metric of Equation 15 was significantly higher for the MCI patient group compared to the matched controls. It has been demonstrated that MCI patients exhibit higher synchronisation in MEG studies [51] and functional MRI studies [52]. The disparity between lower power but higher synchronisation between the patients and controls of the MCI group but not the MCI-FAD group can be explained firstly by noting that the average age of the MCI group is much higher (73) than the MCI-FAD group (44). The Scaffolding Theory of Cognitive Aging and Decline (STCA) [53], [36] describes the compensatory mechanisms that are recruited by the brain to alleviate age related cognitive decline. In memory tasks, MCI patients exhibit increased connectivity between brain areas as aging progresses and compared to controls [36]. This is also shown in this study where increased connectivity is found in the MCI patient group only as compared to the age matched controls due to the larger cognitive decline corroborating the STCA theory. Lastly, the binding task consisted of more distributed brain activations for the binding than the shape task. This is further evidence that the binding tasks requires brain connectivity between different brain areas since the shape and colour binding process invokes separate brain centres [54].

\section{CONCLUSIONS}

In this work we showed that complex tensor factorisation based on complex PARAFAC2 is suitable for EEG connectivity estimation and superior to the complex PARAFAC. By establishing that EEG data follow the PARAFAC2 model in the complex domain a tensor factorisation algorithm was successful in a benchmark and two EEG datasets. As compared to MVAR-ICA, a matrix based source estimation method, PARAFAC2 performed better apart from very low PR values where MVAR-ICA was better at estimating the connectivity of the coupled sources.

This work has the following implications. Firstly, the use of high order methods such as tensor factorisation is suitable 

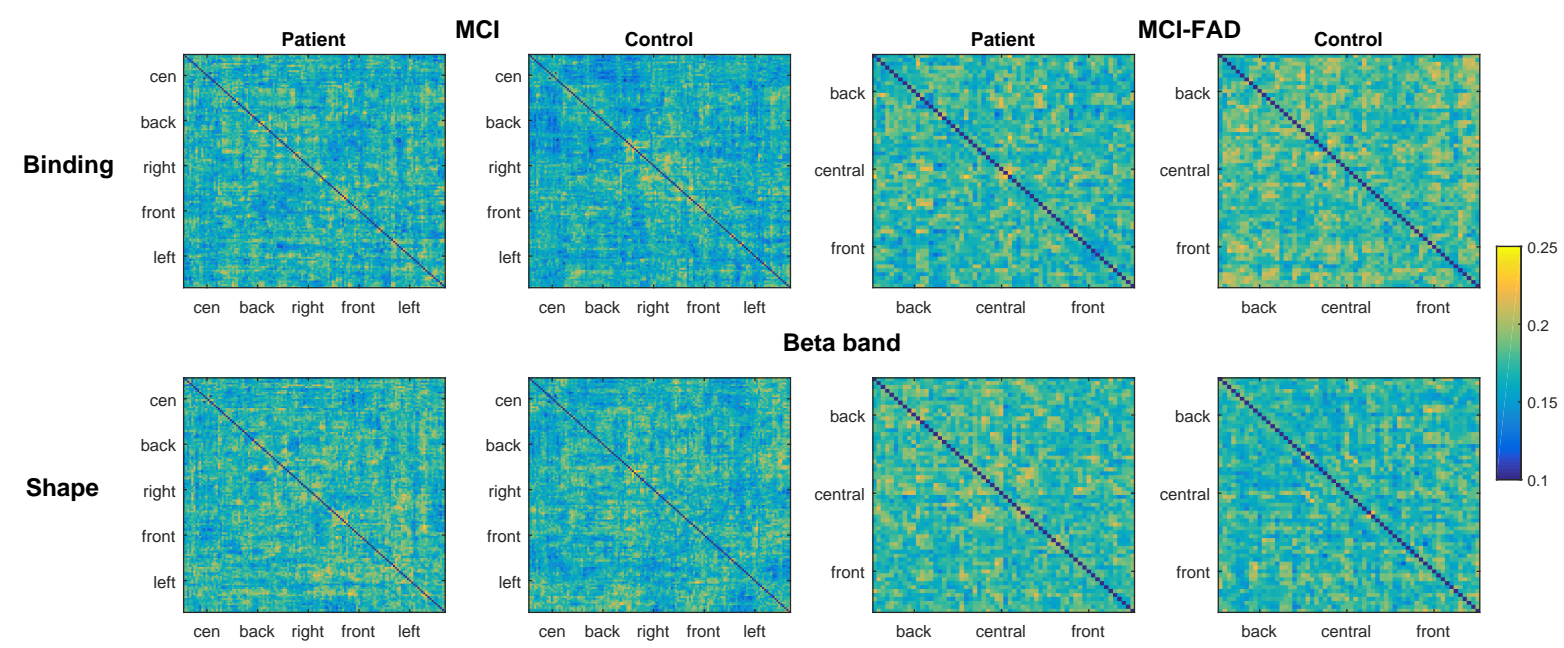

Fig. 11. Connectivity map for the standard PLI measure and the MCI (left) and MCI-FAD (right) datasets in the beta band. Each point indicates the coupling between two electrodes which have been grouped into five regions over the scalp.
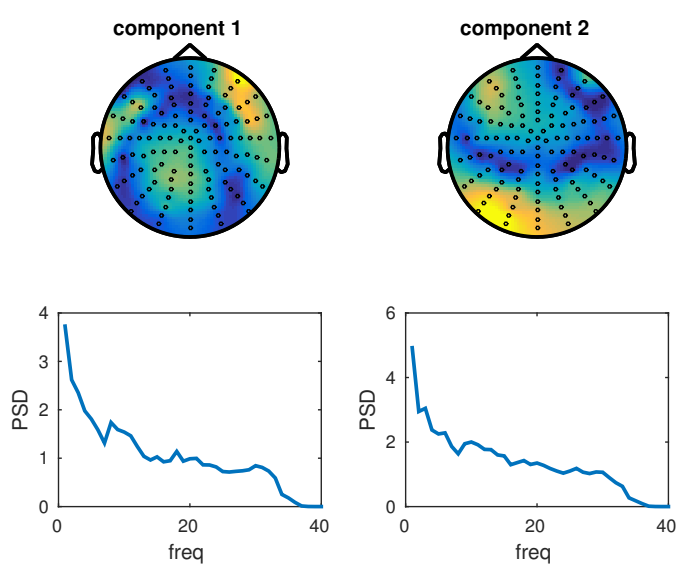

Fig. 12. Example source pair obtained by PARAFAC2. Top row shows the spatial topography of the two sources while the bottom row shows their frequency profiles. These two components had a connectivity strength of 0.25 .

for extracting coupled sources. And secondly, performing tensor factorisation in the complex domain allows for the connectivity information present in the data to be optimally exploited. Importantly, the decoupling of spatial, spectral, and synchronisation behaviour of networks is not possible with traditional connectivity metrics and this work aims to bridge that gap. This works opens up a new possibility in the inspection of EEG connectivity.

Future work entails modification of the PARAFAC2 constraint to better facilitate the EEG data generation model. In that light, different data models will be considered such as phase/non-phase locked, event related potentials, and the presence of multiple coupled source pairs. We also plan to validate the algorithm on a longitudinal $\mathrm{AD}$ dataset of more than 100 patients over 5 years.

\section{ACKNOWLEDGMENTS}

We wish to thank Agustín Ibáñez for the provision of the dataset [47]. 


\section{REFERENCES}

[1] Rasmus Bro, "PARAFAC. Tutorial and applications," in Chemometrics and Intelligent Laboratory Systems, 1997, vol. 38, pp. 149-171.

[2] Richard A. Harshman, "Foundations of the parafac procedure: models and conditions for an explanatory multimodal factor analysis," vol. 16, pp. 1-84, 1970.

[3] Richard A. Harshman, "PARAFAC2: Mathematical and technical notes," UCLA Working Papers in Phonetics, vol. 22, no. 10, pp. 30-44, 1972.

[4] Pierre Comon, "Tensor decompositions, state of the art and applications," arXiv preprint:0905.0454, 2009.

[5] Pieter M. Kroonenberg and Jan De Leeuw, "Principal component analysis of three-mode data by means of alternating least squares algorithms," Psychometrika, vol. 45, no. 1, pp. 69-97, 1980.

[6] Andrzej Cichocki, "Tensor decompositions: a new concept in brain data analysis?," arXiv preprint:1305.0395, 2013.

[7] Henk Kiers, Jos Ten Berge, and Rasmus Bro, "PARAFAC2 Part I . A Direct Fitting Algorithm for the PARAFAC2 Model," J. Chemom., vol. 13, no. July 1998, pp. 275-294, 1999.

[8] Sanei Sanei, Adaptive Processing of Brain Signals, Wiley, 2007.

[9] Evrim Acar, Canan Aykut-Bingol, Haluk Bingol, Rasmus Bro, and Bulent Yener, "Multiway analysis of epilepsy tensors," Bioinformatics, vol. 23, no. 13, pp. i10-i18, 2007.

[10] Hyekyoung Lee, Yong-deok Kim, Andrez Cichocki, and Seungjin Choi, "Nonnegative Tensor Factorization for Continuous Eeg Classification," International Journal of Neural Systems, vol. 17, no. 04, pp. 305-317, 2007.

[11] Loukianos Spyrou, Samaneh Kouchaki, and Sanei Sanei, "Multiview classification of brain data through tensor factorisation," in Proceeding of IEEE Machine Learning for Signal Processing (MLSP). IEEE, 2015, pp. 1-6.

[12] Fengyu Cong, Qiu-Hua Lin, Li-Dan Kuang, Xiao-Feng Gong, Piia Astikainen, and Tapani Ristaniemi, "Tensor decomposition of EEG signals: A brief review," Journal of Neuroscience Methods, vol. 248, pp. 59-69, 2015.

[13] Loukianos Spyrou, Samaneh Kouchaki, and Sanei Saeid, "Multiview classification and dimensionality reduction of scalp and intracranial eeg data through tensor factorisation," Journal of Signal Processing Systems, pp. 1-12, 2016.

[14] Guido Nolte, Ou Bai, Lewis Wheaton, Zoltan Mari, Sherry Vorbach, and Mark Hallett, "Identifying true brain interaction from EEG data using the imaginary part of coherency," Clinical Neurophysiology, vol. 115 no. 10 , pp. 2292-2307, 2004

[15] Stefan Haufe, Ryota Tomioka, Guido Nolte, Klaus Robert Müller, and Motoaki Kawanabe, "Modeling sparse connectivity between underlying brain sources for EEG/MEG," IEEE Transactions on Biomedical Engineering, vol. 57, no. 8, pp. 1954-1963, 2010.

[16] Marcin Jan Kaminski and Katarzyna J. Blinowska, "A new method of the description of the information flow in the brain structures," Biological Cybernetics, vol. 65, no. 3, pp. 203-210, 1991.

[17] Stefan Haufe, Vadim V. Nikulin, Klaus Robert Muller, and Guido Nolte, "A critical assessment of connectivity measures for EEG data: A simulation study," NeuroImage, vol. 64, no. 1, pp. 120-133, 2013.

[18] Frederik van de Steen, Luca Faes, Esin Karahan, Jitkomut Songsiri, Pedro A. Valdes-Sosa, and Daniele Marinazzo, "Critical Comments on EEG Sensor Space Dynamical Connectivity Analysis," Brain Topography, pp. 1-12, 2016.

[19] Cornelis J. Stam, Guido Nolte, and Andreas Daffertshofer, "Phase lag index: Assessment of functional connectivity from multi channel EEG and MEG with diminished bias from common sources," Human Brain Mapping, vol. 28, no. 11, pp. 1178-1193, 2007.

[20] Federico Chella, Vittorio Pizzella, Filippo Zappasodi, and Laura Marzetti, "Impact of the reference choice on scalp eeg connectivity estimation," Journal of Neural Engineering, vol. 13, no. 3, pp. 036016 , 2016.

[21] Clemens Brunner, Martin Billinger, Martin Seeber, Timothy R. Mullen, and Scott Makeig, "Volume Conduction Influences Scalp-Based Connectivity Estimates," Frontiers in Computational Neuroscience, vol. 10, no. November, pp. 121, 2016.

[22] Martin Vinck, Robert Oostenveld, Marijn Van Wingerden, Franscesco Battaglia, and Cyriel M A Pennartz, "An improved index of phasesynchronization for electrophysiological data in the presence of volumeconduction, noise and sample-size bias," NeuroImage, vol. 4, no. 55, pp. 1548-65, 2011.

[23] Anton Sirota, Sean Montgomery, and Shigeyoshi Fujisawa, "Entrainment of neocortical neurons and gamma oscillations by the hippocampal theta rhythm," Neuron, vol. 60, no. 4, pp. 683-697, 2008.
[24] Ramesh Srinivasan, William R. Winter, Jian Ding, and Paul L. Nunez, "EEG and MEG coherence: Measures of functional connectivity at distinct spatial scales of neocortical dynamics," Journal of Neuroscience Methods, vol. 166, no. 1, pp. 41-52, 2007.

[25] Guido Nolte, Andreas Ziehe, Vadim V. Nikulin, Alois Schlögl, Nicole Krämer, Tom Brismar, and Klaus Robert Müller, "Robustly estimating the flow direction of information in complex physical systems," Physical Review Letters, vol. 100, no. 23, pp. 1-4, 2008.

[26] Jean Philippe Lachaux, Eugenio Rodriguez, Jacques Martinerie, and Francisco J. Varela, "Measuring phase synchrony in brain signals," Human Brain Mapping, vol. 8, no. 4, pp. 194-208, 1999.

[27] Martin Vinck, Marijn van Wingerden, Thilo Womelsdorf, Pascal Fries, and Cyriel M.A. Pennartz, "The pairwise phase consistency: A bias-free measure of rhythmic neuronal synchronization," NeuroImage, vol. 51, no. 1 , pp. 112-122, 2010

[28] Germán Gómez-Herrero, Mercedes Atienza, Karen Egiazarian, and Jose L. Cantero, "Measuring directional coupling between EEG sources," NeuroImage, vol. 43, no. 3, pp. 497-508, 2008.

[29] Arash Golibach Mahyari and Selin Aviyente, "Identification of dynamic functional brain network states through tensor decomposition," Acoustics, Speech and Signal Processing (ICASSP), 2014 IEEE International Conference on, pp. 2099-2103, 2014.

[30] Britta Pester, Carolin Ligges, Lutz Leistritz, Herbert Witte, and Karin Schiecke, "Advanced Insights into Functional Brain Connectivity by Combining Tensor Decomposition and Partial Directed Coherence," PLoS One, vol. 10, pp. 10.1371, 2015.

[31] Daniel Abásolo, Roberto Hornero, Javier Escudero, and Pedro Espino, "A study on the possible usefulness of detrended fluctuation analysis of the electroencephalogram background activity in Alzheimer's disease," IEEE Transactions on Biomedical Engineering, vol. 55, no. 9, pp. 21712179,2008

[32] Nora Leonardi and Dimitri Van De Ville, "Identifying network correlates of brain states using tensor decompositions of whole-brain dynamic functional connectivity," Proceedings - 2013 3rd International Workshop on Pattern Recognition in Neuroimaging, PRNI 2013, pp. 74-77, 2013.

[33] Morten Mørup, Lars Kai Hansen, Sidse Marie Arnfred, Lek Heng Lim, and Kristoffer Hougaard Madsen, "Shift-invariant multilinear decomposition of neuroimaging data," NeuroImage, vol. 42, no. 4, pp. 1439-1450, 2008

[34] Roemer van der Meij, Joshua Jacobs, and Eric Maris, "Uncovering phase-coupled oscillatory networks in electrophysiological data," Human Brain Mapping, vol. 36, no. 7, pp. 2655-2680, 2015.

[35] Samaneh Kouchaki, Shirin Enshaeifar, Clive Cheong Took, and Saeid Sanei, "Complex tensor based blind source separation of EEG for tracking P300 subcomponents," Proceedings IEEE EMBC 2015, 2015.

[36] Roberto Cabeza, Nicole D. Anderson, Jill K. Locantore, and Anthony R. McIntosh, "Aging Gracefully: Compensatory Brain Activity in HighPerforming Older Adults," NeuroImage, vol. 17, no. 3, pp. 1394-1402, 2002.

[37] Hans Hallez, Bart Vanrumste, Roberta Grech, Joseph Muscat, Wim De Clercq, Anneleen Vergult, Yves D'Asseler, Kenneth P. Camilleri, Simon G. Fabri, Sabine Van Huffel, and Ignace Lemahieu, "Review on solving the forward problem in eeg source analysis," Journal of NeuroEngineering and Rehabilitation, vol. 4, no. 1, pp. 46, Nov 2007.

[38] John C. Mosher, Richard M. Leahy, and Paul S. Lewis, "EEG and MEG: forward solutions for inverse methods," IEEE Transactions on Biomedical Engineering, vol. 46, no. 3, pp. 245-259, 1999.

[39] Christoph M. Michel, Micah M. Murray, Gran Lantz, Sara Gonzalez, Laurent Spinelli, and Rolando Grave de Peralta, "EEG source imaging.," Clinical neurophysiology, vol. 115, no. 10, pp. 2195-222, Oct. 2004.

[40] Loukianos Spyrou and Saeid Sanei, "Source localization of event-related potentials incorporating spatial notch filters," IEEE Transactions on Biomedical Engineering, vol. 55, no. 9, pp. 2232-2239, 2008.

[41] James Pardey, Stephen Roberts, and Lionel Tarassenko, "A review of parametric modelling techniques for EEG analysis," Medical Engineering and Physics, vol. 18, no. 1, pp. 2-11, 1996.

[42] Stefan Haufe and Arne Ewald, "A Simulation Framework for Benchmarking EEG-Based Brain Connectivity Estimation Methodologies," Brain Topography, jun 2016.

[43] "Mechanisms of evoked and induced responses in MEG/EEG," NeuroImage, vol. 31, no. 4, pp. 1580-1591, 2006.

[44] Matteo Fraschini, Matteo Demuru, Alessandra Crobe, Francesco Marrosu, Cornelius Stam, and Arjan Hillebrand, "The effect of epoch length on estimated EEG functional connectivity and brain network organisation," Journal of Neural Engineering, vol. 13, no. 3, pp. 036015 , 2016. 
[45] Shinya Kasakawa, Teruya Yamanishi, Tetsuya Takahashi, Kanji Ueno, Mitsuru Kikuchi, and Haruhiko Nishimura, Approaches of Phase Lag Index to EEG Signals in Alzheimer's Disease from Complex Network Analysis, pp. 459-468, Springer International Publishing, Cham, 2016.

[46] Maja H. Kamstrup-Nielsen, Lea G. Johnsen, and Rasmus Bro, "Core consistency diagnostic in PARAFAC2," Journal of Chemometrics, vol. 27, no. 5, pp. 99-105, 2013.

[47] Marcos Pietto, Mario Parra, Natalia Trujillo, Facundo Flores, Adolfo Garca, Julian Bustin, Pablo Richly, Facundo Manes, Francisco Lopera, Agustin Ibez, and Sandra Baez, "Behavioral and Electrophysiological Correlates of Memory Binding Deficits in Patients at Different Risk Levels for Alzheimers Disease," Journal of Alzheimer's Disease, vol. 53, pp. 1325-1340, 2016.

[48] Mario A. Parra, Sharon Abrahams, Robert H. Logie, and Sergio Della Sala, "Visual short-term memory binding in Alzheimer's disease and depression," Journal of Neurology, vol. 257, no. 7, pp. 1160-1169, 2010.

[49] Elham Barzegaran and Maria G. Knyazeva, "Functional connectivity analysis in EEG source space: The choice of method," PLOS ONE, vol. 12, no. 7, pp. 1-16, 2017.

[50] Rasmus Bro, Claus Anderson, and Henk Kiers, "PARAFAC2 Part II. Modeling chromatographic data with retention time shifts," Journal of Chemometrics, vol. 13, no. 3-4, pp. 295-309, 1999.

[51] Javier M. Buldú, Ricardo Bajo, Fernando Maestú, Nazareth Castellanos, Inmaculada Leyva, Pablo Gil, Irene Sendiña-Nadal, Juan A. Almendral, Angel Nevado, Francisco Del-Pozo, and Stefano Boccaletti, "Reorganization of functional networks in mild cognitive impairment," PLOS ONE, vol. 6, no. 5, 2011.

[52] Bradford Dickerson, "Increased hippocampal activation in mild cognitive impairment compared to normal aging and AD," Biophysical Chemistry, vol. 257, no. 5, pp. 2432-2437, 2005.

[53] Joshua Goh and D.C. Park, "Neuroplasticity and cognitive aging: The scaffolding theory of aging and cognition," Restor Neurol Neurosci, vol. 27, no. 5, pp. 391-403, 2009.

[54] Mario A. Parra, Sergio Della Sala, Robert H. Logie, and Alexa M. Morcom, "Neural correlates of shape-color binding in visual working memory," Neuropsychologia, vol. 52, no. 1, pp. 27-36, 2014. 